\title{
EXPERIMENTAL DEMONSTRATION OF MICROWAVE SIGNAL/ELECTRIC THRUSTER PLASMA INTERACTION EFFECTS
}

\author{
Afroz J. Zaman "1, Kevin M. Lambert ${ }^{2}$, Frank M. Curran' \\ 'NASA Lewis Research Center \\ 21000 Brookpark Road, M/S 54-8 \\ Cleveland, Ohio 44135 \\ Phone: $216-433-3415$ \\ FAX: 216-433-8705
}

and

${ }^{2}$ ANALEX Corporation

3001 Aerospace Parkway

Brook Park, Ohio 44142

Phone: 216-433-6388

FAX: 216-433-3344

\begin{abstract}
An experiment was designed and conducted in the Electric Propulsion Laboratory of NASA Lewis Research Center to assess the impact of ion thruster exhaust plasma plume on electromagnetic signal propagation.. A microwave transmission experiment was set up inside the propulsion test bed using a pair of broadband hom antennas and a $30 \mathrm{~cm} 2.3 \mathrm{~kW}$ ion thruster. Frequency of signal propagation covered from 6.5 to $18 \mathrm{GHz}$ range. The stainless steel test bed when enclosed can be depressurized to simulate a near vacuum environment. A pulsed CW system with gating hardware was utilized to eliminate multiple chamber reflections from the test signal. Microwave signal was transmitted and received between the two homs when the thruster was operating at a given power level in such a way that the signal propagation path crossed directly through the plume volume. Signal attenuation and phase shift due to the plume was measured for the entire frequency band. Results for this worst case configuration simulation indicate that the effects of the ion thruster plume on microwave signals is a negligible attenuation ( within $0.15 \mathrm{~dB}$ ) and a small phase shift (within $8^{0}$ ). This paper describes the detailed experiment and presents some of the results.
\end{abstract}

\section{INTRODUCTION}

Electric thruster, a high performance propulsion technology is currently being considered for a wide variety of space missions, such as; insertion and orbital maintenance of SmallSats, compensation of on orbit drifts , drag compensation etc., because of the promise it offers to fill a wide range of propulsion needs. The main advantage of using electric propulsion is that the electric energy added to the exhaust propellant greatly increases its velocity, or specific impulse; and hence the mass of propellant required to produce a given thrust decreases greatly or more thrust is produced with the same propellant flow rate. This significant propellant mass savings impacts favorably in several areas such as launch vehicle size, satellite lifetime and mission payload. The impact of electric thruster plasma plumes on the performance of on-board spacecraft antennas however, is a primary integration concern for 
planners of the next generation spacecrafts considering electric propulsion. Figure 1 depicts a potential impact scenario on board a spacecraft. Arcjets were the first high performance electrothermal thrusters to be considered for operational missions. The impact of arcjet plasma plumes on microwave signals was analyzed and modeled by Ling, et.al.[1]. The model was subsequently used by Lockheed Martin Astro Space for specific case studies of the series 7000 geosynchronous communications satellite. The results predicted the weakly ionized plumes from arcjets would have a negligible effect on communication signals and led arcjets to obtain operational status since the early 1990's. State-ofthe-art arcjets using hydrazine propellant with a specific impulse of about 600 seconds are to be launched in commercial satellites sometime around the tum of the century. Ion engines using xenon as propellant can produce specific impulse ranging from 3000 seconds and above. These types of thrusters will be used for north-south stationkeeping on the upcoming Hughes 600 and 700 series satellites. In addition, ion thruster being developed under NSTAR (NASA Solar electric propulsion Technology Application Readiness) program was recently baselined for use on the firșt New Millennium spacecraft to be launched in 1998 for primary propulsion in an ambitious planetary mission. However, Ling's model will not likely be sufficient for predicting the impact due to the high performance electric thrusters like the ion and Hall thrusters that exhibit higher ionization levels, plasma temperatures and particle velocities than arcjets. To address this need the authors have designed and performed an experiment to assess the attenuation and phase shift caused by these types of plasmas on microwave signals in the $6.5-18 \mathrm{GHz}$ frequency band. The main challenge in performing a propagation experiment in a simulated space environment is introduced by the multiple wall reflections that are created inside the conducting chamber. Thus the experiment had to be designed to eliminate unwanted wall reflections from the test signals. This paper describes the experimental technique that was developed to minimize the effects of the reflections and presents some of the results that were obtained.

\section{ELECTRIC PROPULSIONSYSTEM}

Historically all propulsion system for planetary or Earth orbital applications have been chemical devices. However for applications where low accelerations are acceptable or desirable and where fuel efficiency is a mission driver, electric propulsion can make a significant impact. Electric propulsion can provide high propellant velocity or increased fuel efficiency at a reduced thrust and is useful only for in-space applications. Nearly every space mission requires on-board propulsion systems and these systems are often dominant spacecraft mass drivers. This is true both for traditional spacecraft such as large geosynchronous communications satellites and for the spacecraft being designed for distributed lowand mid-Earth orbital communications systems, remote sensing, and space science missions. Presently, on-board systems account for more than half the injected mass for commercial communications systems and even greater mass fractions for ambitious planetary missions. Electric thrusters can provide significant fuel economies as compared to their chemical counterparts (up to an order of magnitude depending on the system and application ). The acceptance of arcjet thrusters for operational use on commercial communications satellites sets a new stage for electric thrusters and growth of electric propulsion across a broad spectrum of missions is expected. It is anticipated that advanced electric propulsion systems will be an option to perform auxiliary propulsion functions like autonomous stationkeeping, orbit period control, drag cancellation etc. for GEO, LEO/MEO spacecrafts in the near-term. Several primary functions of electric propulsion currently under study includes earth orbit transfer, apogee assist and planetary exploration.

There are three classes of electric propulsion concepts; electrothermal, where propellant is heated by resistors or arcs and expanded through a nozzle; electrostatic, where ionized propellant is accelerated by electrostatic means; and electromagnetic, where plasmas are accelerated by electric and magnetic fields.

An ion thruster, shown in figure 2, is an electrostatic propulsion system and uses xenon as propellant. The 
principal elements of a ion thruster propulsion system includes solar cells that converts solar energy into electric power, power conditioner, that produces current and voltage needed by the thruster, discharge chamber where gaseous propellant is ionized by electron bombardment and then accelerated out as ion beam by an electric field. Exhaust propellant is nearly 100 percent ionized; its velocity is in thousands of $\mathrm{km} / \mathrm{sec}$ and plasma temperature is in the order of $2 \mathrm{eV}$.

\section{DESCRIPTION OF THE EXPERIMENT}

The experiments were performed in the Electric Propulsion Laboratory of the NASA Lewis Research Center. The laboratory test bed utilizes a cylindrical stainless steel vacuum chamber of $5 \mathrm{~m}$ diameter and $20 \mathrm{~m}$ length. The test bed is equipped to make diagnostic and integration measurements of propulsion devices in a simulated space environment. The vacuum capability of this facility can range up to $10^{-7}$ Torr and is achieved in three stages of pumping that includes diffusion pumps and a cryogenic panel. The equipments required to perform the propagation experiment were recently installed. For the experiment described here, a $2.3 \mathrm{~kW}, 30 \mathrm{~cm}$ ion thruster was mounted along the axial line of the chamber, near one of the endcaps of the cylinder. A pair of broadband horn antennas were mounted inside the vacuum chamber, near the side walls, such that microwave signal propagation path between the antennas passed directly through the plasma plume of the thruster. The thruster was mounted on a movable cart that allowed measurements to be taken as a function of distance from the origin of the plume. This was done in order to examine the effects of transmission through different ion densities of the plume. Figure 3 is a photograph of the experimental set up inside the vacuum chamber showing the hom antennas on the side walls and the thruster on a movable cart over a platform.

In order to mitigate the effects of wall reflections a pulsed CW system shown in Figure 4 was used. This system based on an 8510 Network Analyzer, uses external hardware time gating to eliminate the clutter resulting from reflections within the vacuum chamber [2]. Since electromagnetic signal travels at the rate of 1 foot per $10^{-9}$ seconds, the signal travel time across the radiating hom antennas was approximately 13 nanoseconds. The $\mathrm{CW}$ transmit signal was pulse modulated in 14 nanosecond width bursts by a pulse controlling switching circuit at the transmit end and was detected by a receiving circuit with a 10 nanosecond gate. Pulse timing and repetition frequency was controlled such that only the test signal was received and all wall reflections were gated out at the receive end. Any remaining error term due to the chamber reflections were minimized through the use of the high gain horn antennas. The signal frequency covered was from 6.5 to $18 \mathrm{GHz}$. with data being taken every $8 \mathrm{MHZ}$. At every frequency point, 64 measurements were taken and averaged to minimize random errors within the measurement system. A single measurement across the frequency band involved 88,000 pulses to be transmitted and received and needed approximately 2 minutes to complete. It was noticed that due to the loss occurring in the 40 feet long cable leading to the transmit pulse modulator, signal power was getting significantly attenuated at the higher frequencies. To cope with this problem, frequency scan range was separated into two bands; one from 6.5 to $12 \mathrm{GHz}$. and the other from 12 to 18 $\mathrm{GHz}$. For the 6.5 to $12 \mathrm{GHz}$ band a $40 \mathrm{~dB}$ of attenuation was inserted in the received signal path before feeding into the test port of the network analyzer. For the 12 to $18 \mathrm{GHz}$ band the attenuation value was lowered to $20 \mathrm{~dB}$. This attenuation was required to avoid setting the high gain amplifiers that are integral with the receive side of the pulse modulator into saturation. A pair of measurements were taken for each thruster condition. First a reference level was established by taking a measurement for the entire frequency band with the thruster off. A second measurement was taken while the thruster was being operated at the desired test conditions. The amplitude and phase of the received signal was recorded as a function of frequency and compared relative to its value at thruster off condition.

\section{RESULTS}

The results from the experiments were produced by 
submitting the data from each pair of measurements to a computer program. The program calculated the amplitude and phase difference of the signal received with the plume on, relative to that of the reference signal, and then plotted the result. Figure 5 shows the signal attenuation and phase shift as a function of frequency for the 6.5 to $12 \mathrm{GHz}$ band for various thruster power levels when the signal propagation path was at a distance $0.5 \mathrm{~m}$ away from the thruster exit plane. As displayed in the plots, each measurement was performed numerous times. This was done in order to determine the repeatability of the tests. Repeatability was a concern because of the small changes being observed. The results indicate an overall insignificant signal attenuation through the plume though this effect increases slightly for increased thruster power levels. This result was anticipated because it is known that the electron density of the plume increases with thruster power. The attenuation effect diminishes at higher frequencies. This was anticipated since the electrical size of the plasma dielectric medium shrinks (though its physical size remains the same) as the frequency increases and as such, the signal experiences less of a dielectric boundary at the same physical interface. The results also indicate a definite phase shift for various thruster power levels. This plot reveals a phase lead which is a function of thruster power, but also becomes negligible as frequency increases. At $0.5 \mathrm{~m}$ from the thruster exit plane and at $2.3 \mathrm{~kW}$ power level, maximum recorded signal attenuation and phase shift was about $0.15 \mathrm{~dB}$ and $8^{\circ}$ respectively. For distances further away from the origin of the plume the magnitude of the effects diminishes considerably although the shape of the plots remains unchanged as is evident from figures 6 and 7.

Figures 8,9 and 10 show the signal attenuation and phase shift as a function of frequency for various thruster power levels for the 12 to $18 \mathrm{GHz}$ frequency band at distances $0.5 \mathrm{~m}, 1.0 \mathrm{~m}$ and $1.5 \mathrm{~m}$ away from the thruster exit plane. These plots display similar overall behavior as mentioned earlier. But at these high frequencies loss in the flexible co-ax cable becomes appreciable and so the ability of the system to detect small changes got impaired and measured attenuation was in effect the system noise. An additional factor that attributed to the cable loss was the ambient temperature during the experimentation. The inside temperature of the laboratory as measured by a thermocouple recorded above $100^{\circ} \mathrm{F}$ during July of 1995 when this experiment was conducted and this factor certainly added to the system noise floor. For the purpose of comparison, results from an earlier proof-of-concept experiment conducted in February of 1994 can be observed [3]. The plots of signal attenuation and phase shift over the entire 6.5 to $18 \mathrm{GHz}$ band displayed almost identical behavior as observed in the present experiment but no appreciable system noise was evident .

In summary, this paper presents the results of an experiment performed at NASA Lewis Research Center to make a quantitative assessment of the impact of high performance electric thruster plasma plumes on microwave signal communication. The results for a worst case configuration simulation conclude that the ion thruster plume introduces insignificant attenuation and very little phase shift to a microwave signal traveling through plume volume.

\section{REFERENCES}

[1] H. Ling, H.Kim, G.A.Hallock, B.W.Birkner, and A.J.Zaman, "Effects of an Arcjet Plume on Satellite Reflector Performance," IEEE Transaction on Antennas and Propagation, vol. 39, no. 9, pp. 1412-1420, September 1991.

[2] T. Barnum, L. Beard, W. Bumside, A. Dominek, W. Lin and E. Walton, "Operating Manual for a pulsed-CW Electromagnetic Transmitter/Receiver and Associated Processing Software," Technical Report 720964-13, The Ohio State University Electro Science Laboratory, February 1991.

[3] K. M. Lambert, A. J. Zaman, and F. M. Curran, "Experimental Demonstration of the Effects of an Electric Thruster Plasma Plume on Microwave Propagation," IEEE AP-S Symposium Digest, vol. 4, pp. 17341737, June 1995. 


\section{Potential Microwave Signal/Plasma Interaction Problem on Board a Satellite}

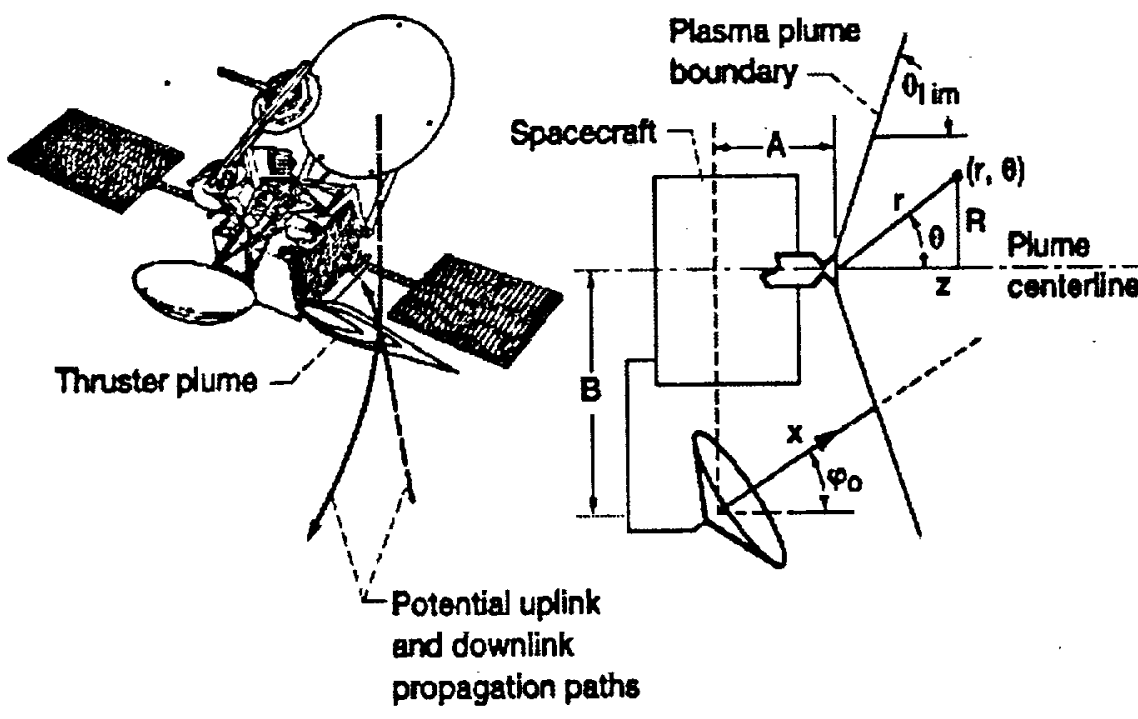

Figure 1. Potential spacecraft antenna/electric thruster plume interaction problem.

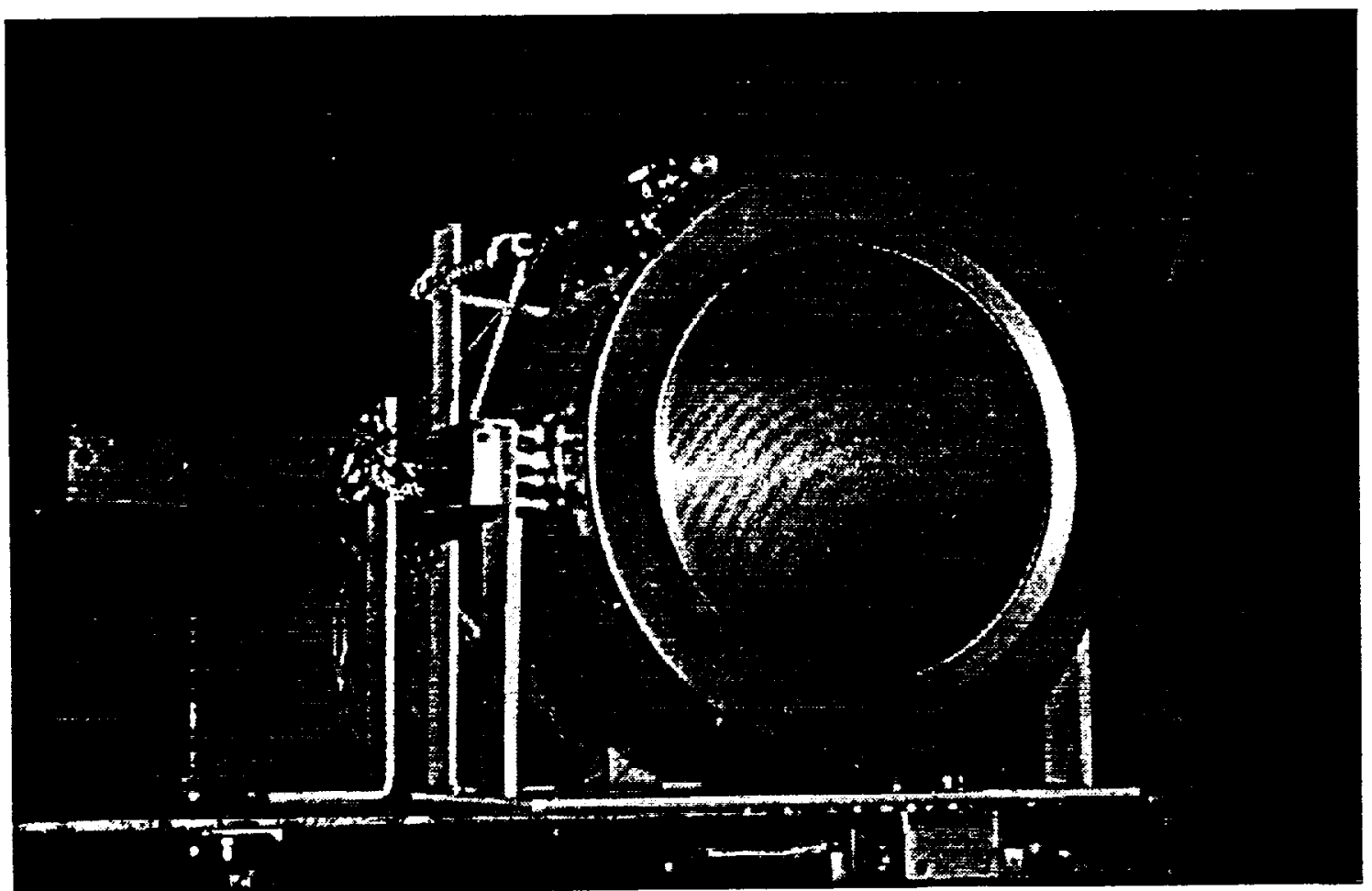

Figure 2. High Performance ( $3000 \mathrm{Isp}$ ) ion thruster for next generation communications satellites. 


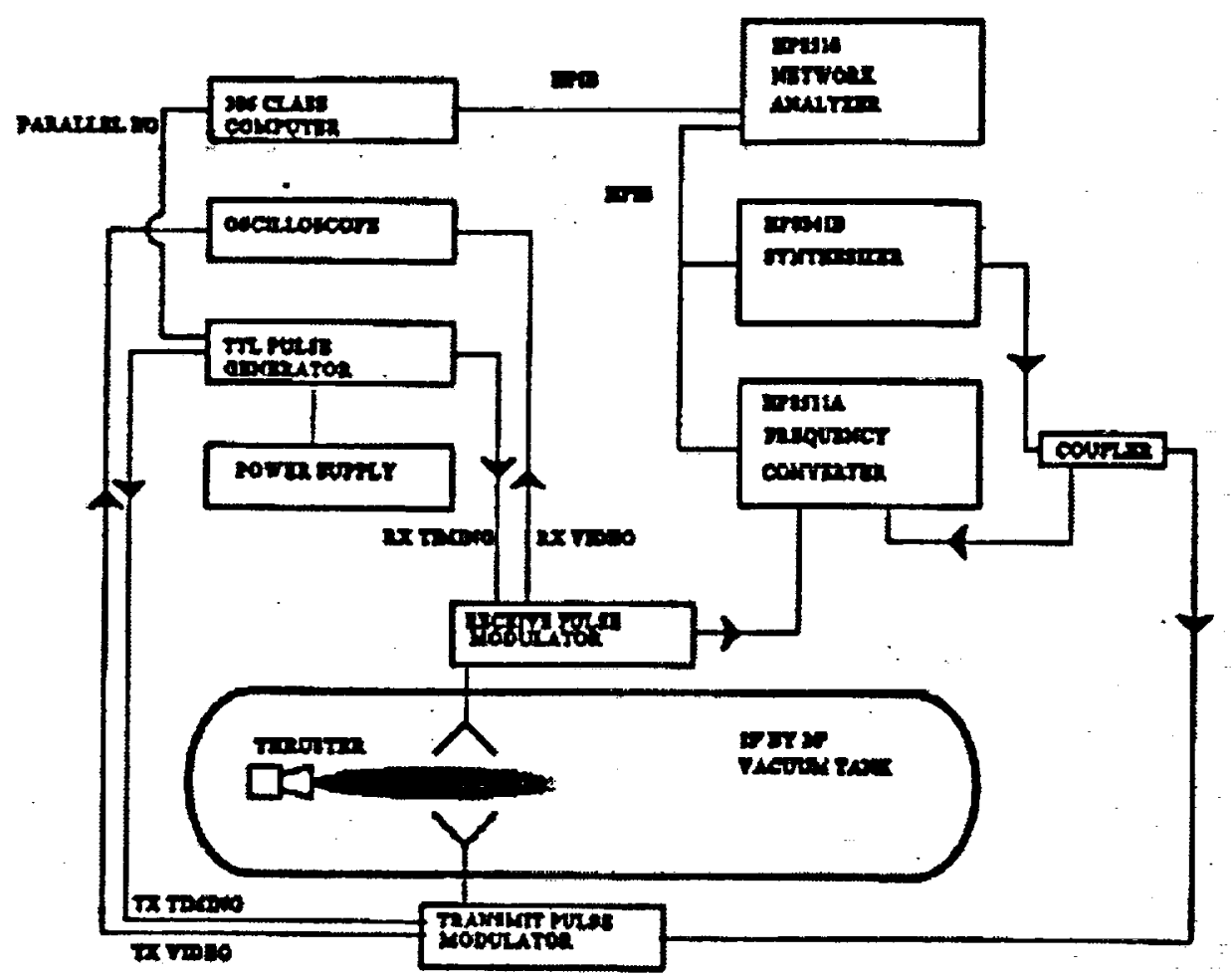

Figure 3. Equipment configuration for the microwave signal/plasma interaction experiment.

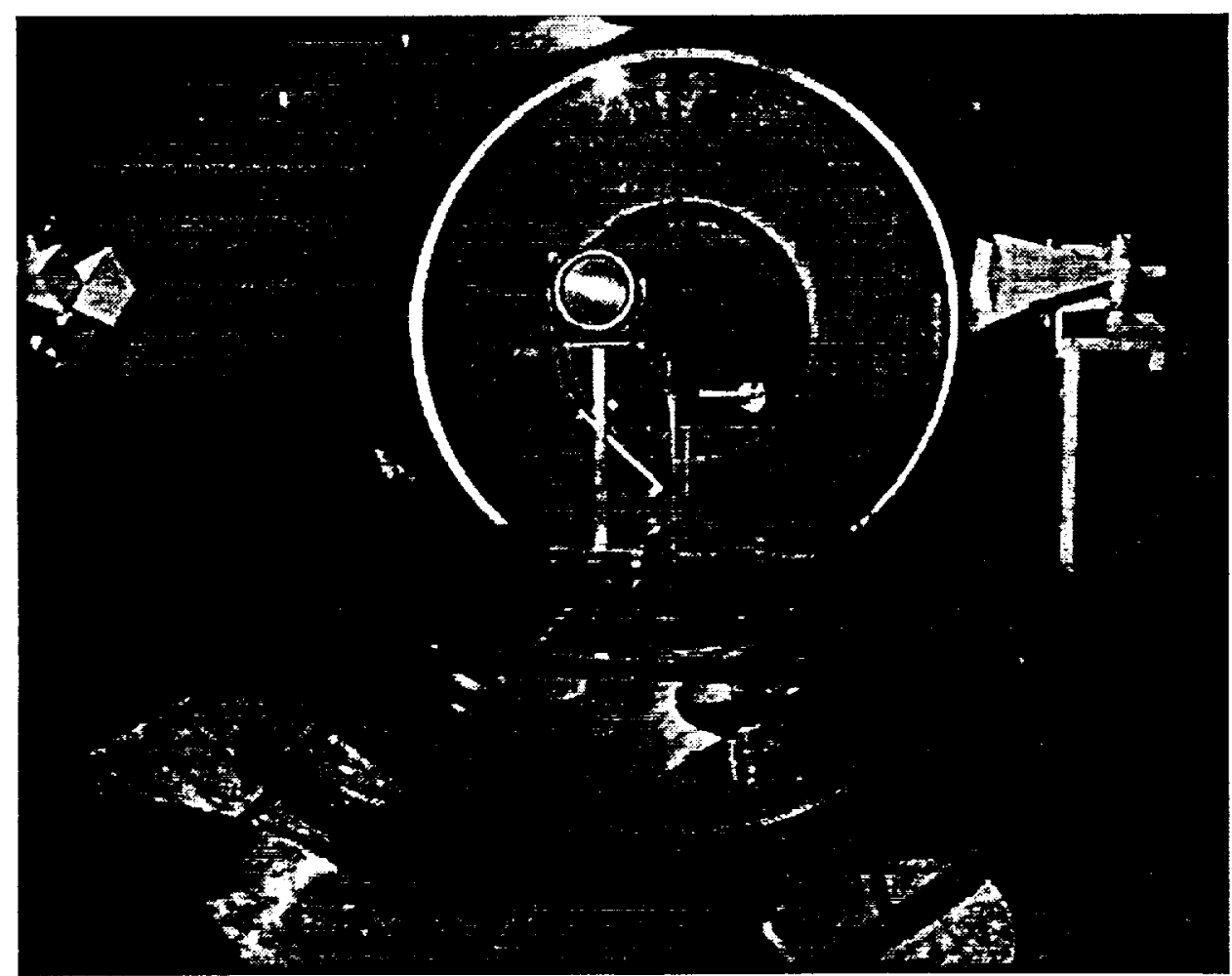

Figure 4. Photograph of the experimental setup inside the test chamber. 

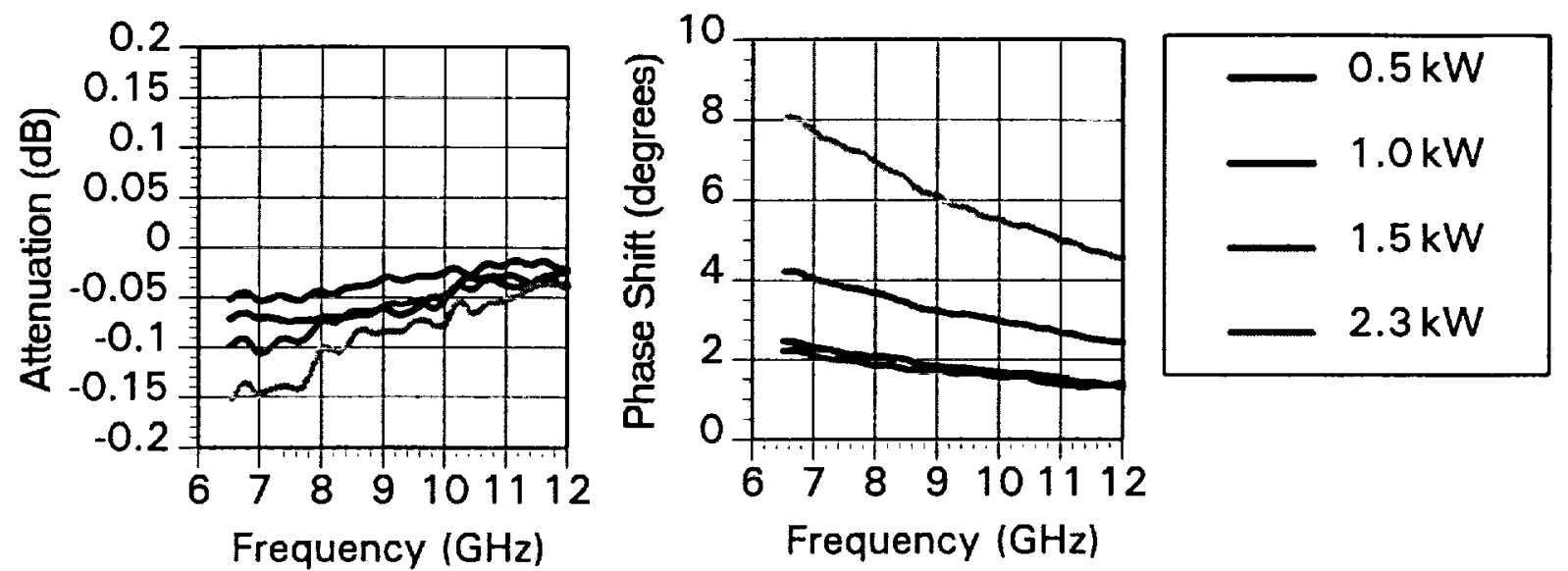

Figure 5. Signal attenuation and phase shift at 0.5 meter from the exit plane.
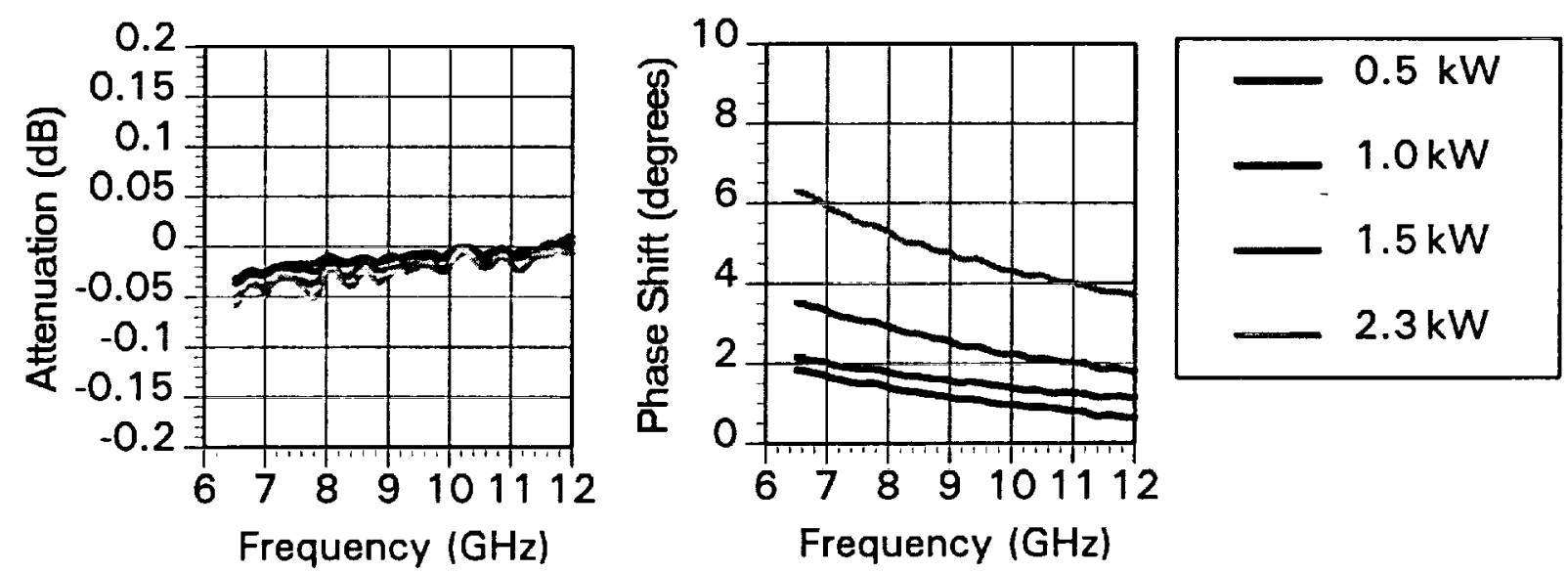

Figure 6. Signal attenuation and phase shift at 1.0 meter from the exit plane.
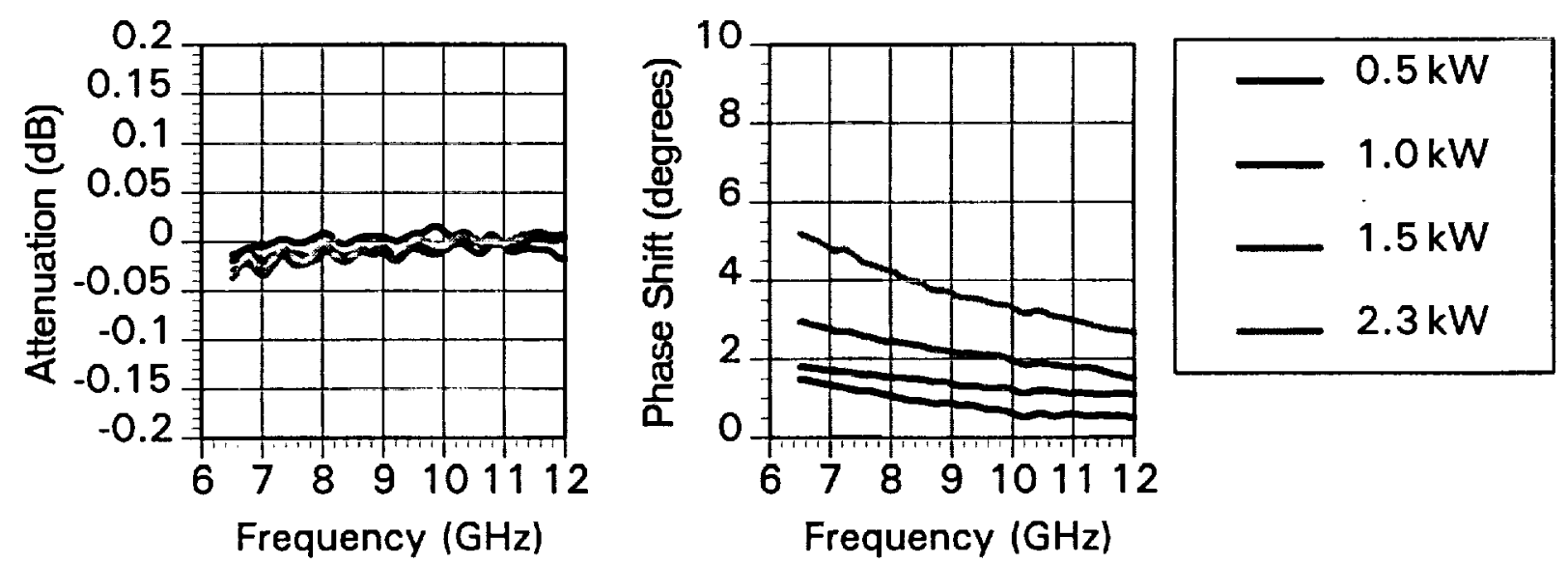

Figure 7. Signal attenuation and phase shift at 1.5 meter from the exit plane. 

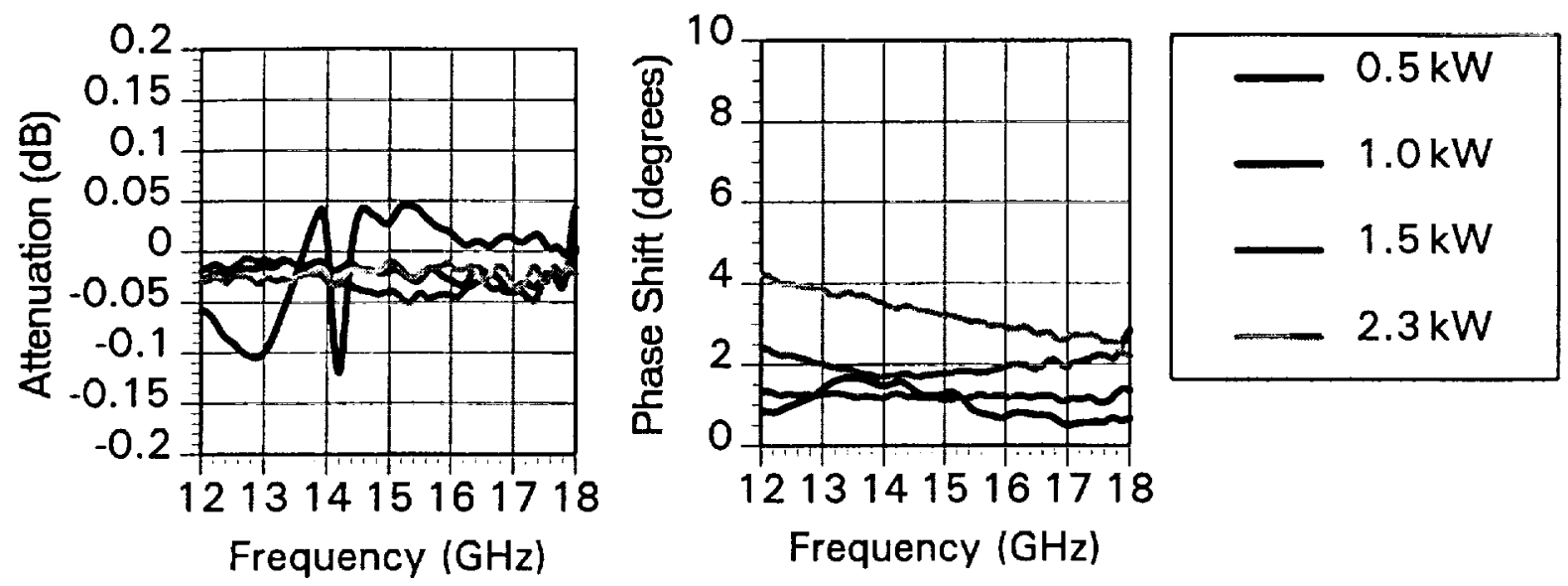

Figure 8. Signal attenuation and phase shift at 0.5 meter from the exit plane.
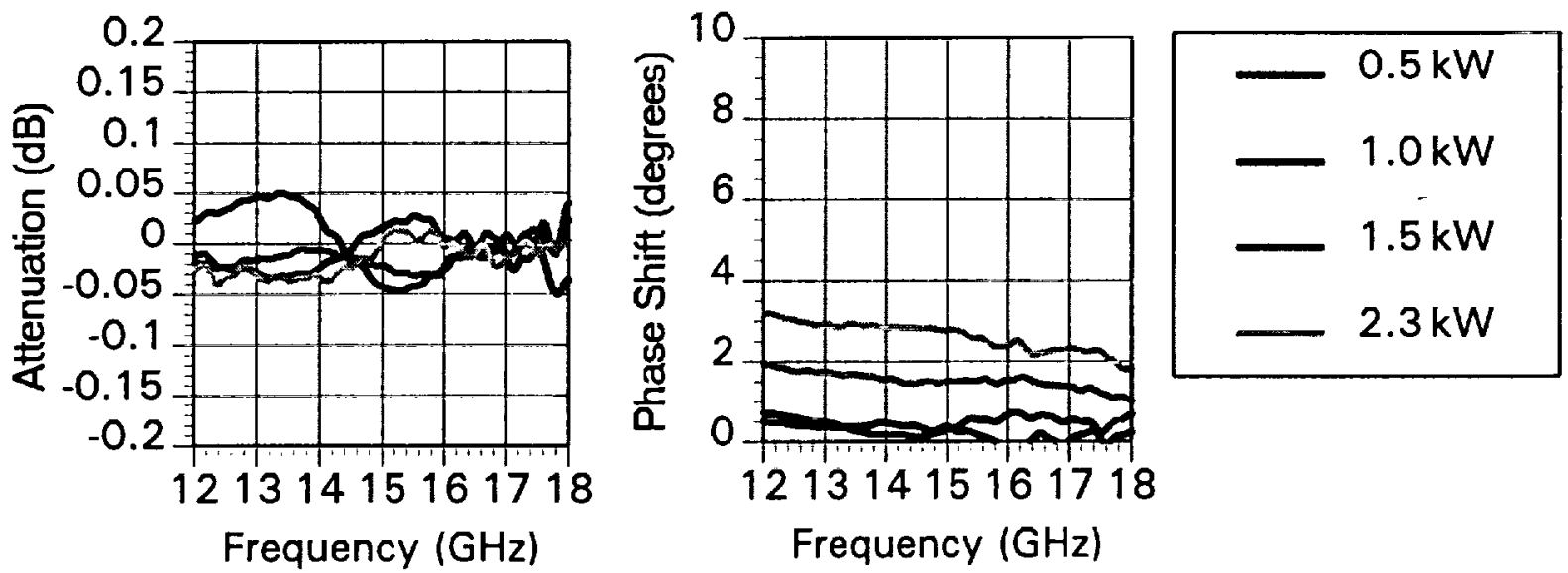

Figure 9. Signal attenuation and phase shift at 1.0 meter from the exit plane.
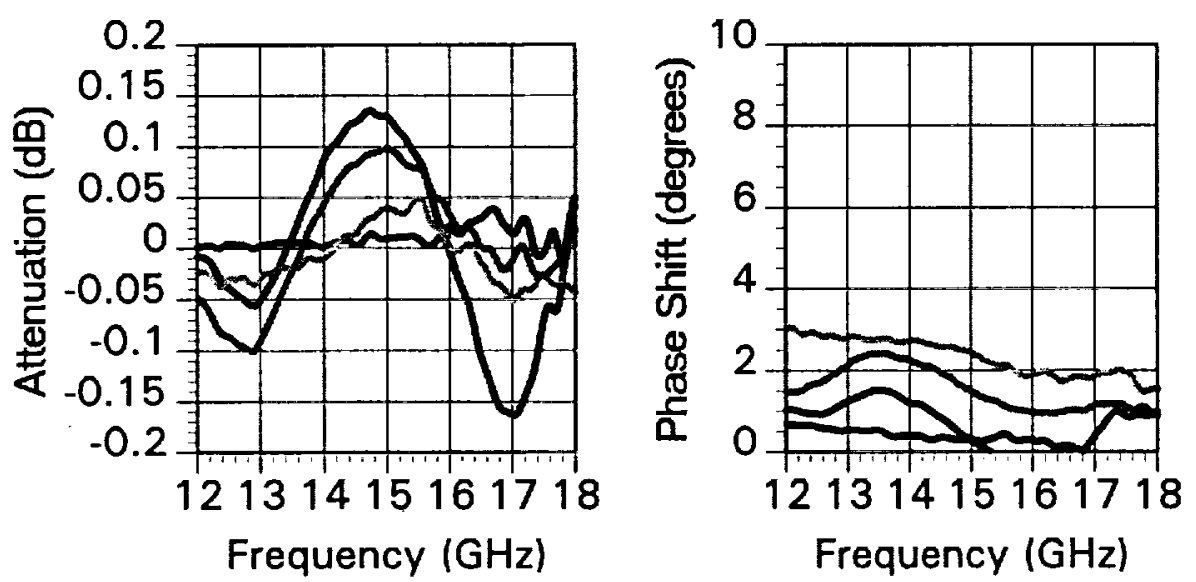

Figure 10. Signal attenuation and phase shift at 1.5 meter from the exit plane. 